\title{
Implications of the semigeostrophic nature of Rossby waves for Rossby wave packet detection
}

Article

Published Version

Wolf, G. and Wirth, V. (2015) Implications of the semigeostrophic nature of Rossby waves for Rossby wave packet detection. Monthly Weather Review, 143 (1). pp. 26-38. ISSN 1520-0493 doi: https://doi.org/10.1175/MWR-D-1400120.1 Available at http://centaur.reading.ac.uk/69071/

It is advisable to refer to the publisher's version if you intend to cite from the work. See Guidance on citing.

Published version at: http://dx.doi.org/10.1175/MWR-D-14-00120.1

To link to this article DOI: http://dx.doi.org/10.1175/MWR-D-14-00120.1

Publisher: American Meteorological Society

All outputs in CentAUR are protected by Intellectual Property Rights law, including copyright law. Copyright and IPR is retained by the creators or other copyright holders. Terms and conditions for use of this material are defined in the End User Agreement. 
CentAUR

Central Archive at the University of Reading

Reading's research outputs online 


\title{
Implications of the Semigeostrophic Nature of Rossby Waves for Rossby Wave Packet Detection
}

\author{
GABRIEL WOLF AND VOLKMAR WIRTH \\ University of Mainz, Mainz, Germany
}

(Manuscript received 4 April 2014, in final form 1 October 2014)

\begin{abstract}
Upper-tropospheric Rossby wave packets have received increased attention recently. In most previous studies wave packets have been detected by computing the envelope of the meridional wind field using either complex demodulation or a Hilbert transform. The latter requires fewer choices to be made and appears, therefore, preferable. However, the Hilbert transform is fraught with a significant problem, namely, a tendency that fragments a single wave packet into several parts. The problem arises because Rossby wave packets show substantial deviations from the almost-plane wave paradigm, a feature that is well represented by semigeostrophic dynamics. As a consequence, higher harmonics interfere with the reconstruction of the wave envelope leading to undesirable wiggles. A possible cure lies in additional smoothing (e.g., by means of a filter) or resorting to complex demodulation (which implies smoothing, too). Another possibility, which does not imply any smoothing, lies in applying the Hilbert transform in semigeostrophic coordinate space. It turns out beneficial to exclude planetary-scale wavenumbers from this transformation in order to avoid problems in cases when the wave packet travels on a low wavenumber quasi-stationary background flow.
\end{abstract}

\section{Introduction}

The statistics of midlatitude weather systems show significant deviations from zonal symmetry, especially in the Northern Hemisphere. This zonal asymmetry is at least partly caused by the uneven distribution of continentalscale orography and land-sea distribution (e.g., Swanson 2007). Close to the surface the asymmetry is associated with a zonal variation of storm tracks (Hoskins and Valdes 1990; Chang and Orlanski 1993). In the upper troposphere it leads to a zonal modulation of Rossby wave amplitudes, giving rise to so-called wave packets or wave trains (Lee and Held 1993; Chang and Yu 1999; Chang 1999). Such wave packets are dynamically relevant as they are associated with zonal (in particular downstream) transfer of energy and momentum (Chang 1993). This may lead to localized downstream effects like surface cyclogenesis (Chang 2005; Wirth and Eichhorn 2014) and severe weather events (Martius et al. 2008; Shapiro and Thorpe 2004). The latter provided the motivation for

Corresponding author address: Dr. Volkmar Wirth, Institute for Atmospheric Physics, University of Mainz, Becherweg 21, 55099 Mainz, Germany.

E-mail: vwirth@uni-mainz.de recent studies on upper-tropospheric Rossby wave packets (Glatt et al. 2011; Glatt and Wirth 2014).

A straightforward method to diagnose Rossby wave packets is based on the zonal variation of the meridional wind $v$. Generally, a wave packet is defined as

$$
v(\lambda)=A(\lambda) C(\lambda),
$$

where $\lambda$ is longitude, $C$ is the so-called carrier wave, and $A$ is the slowly varying amplitude. The amplitude function is nonnegative everywhere and is spatially localized; it has a maximum in the center of the wave packet and smoothly decays to smaller values at the boundaries of the wave packet. The carrier wave $C$ oscillates between positive and negative values and varies on a much shorter spatial scale than $A$. The amplitude $A$ will also be referred to as envelope in the following. The task of envelope reconstruction is tantamount as to find an algorithm that allows one to compute $A(\lambda)$ when $v(\lambda)$ is given. In the past, meteorologists have used essentially two methods in order to reach this goal: complex demodulation (e.g., Lee and Held 1993; Chang and Yu 1999) and the Hilbert transform (Zimin et al. 2003).

Commonly it is assumed that the perturbation has the form of an almost-plane wave, that is, $C(\lambda)=\cos (s \lambda)$ and, hence, 

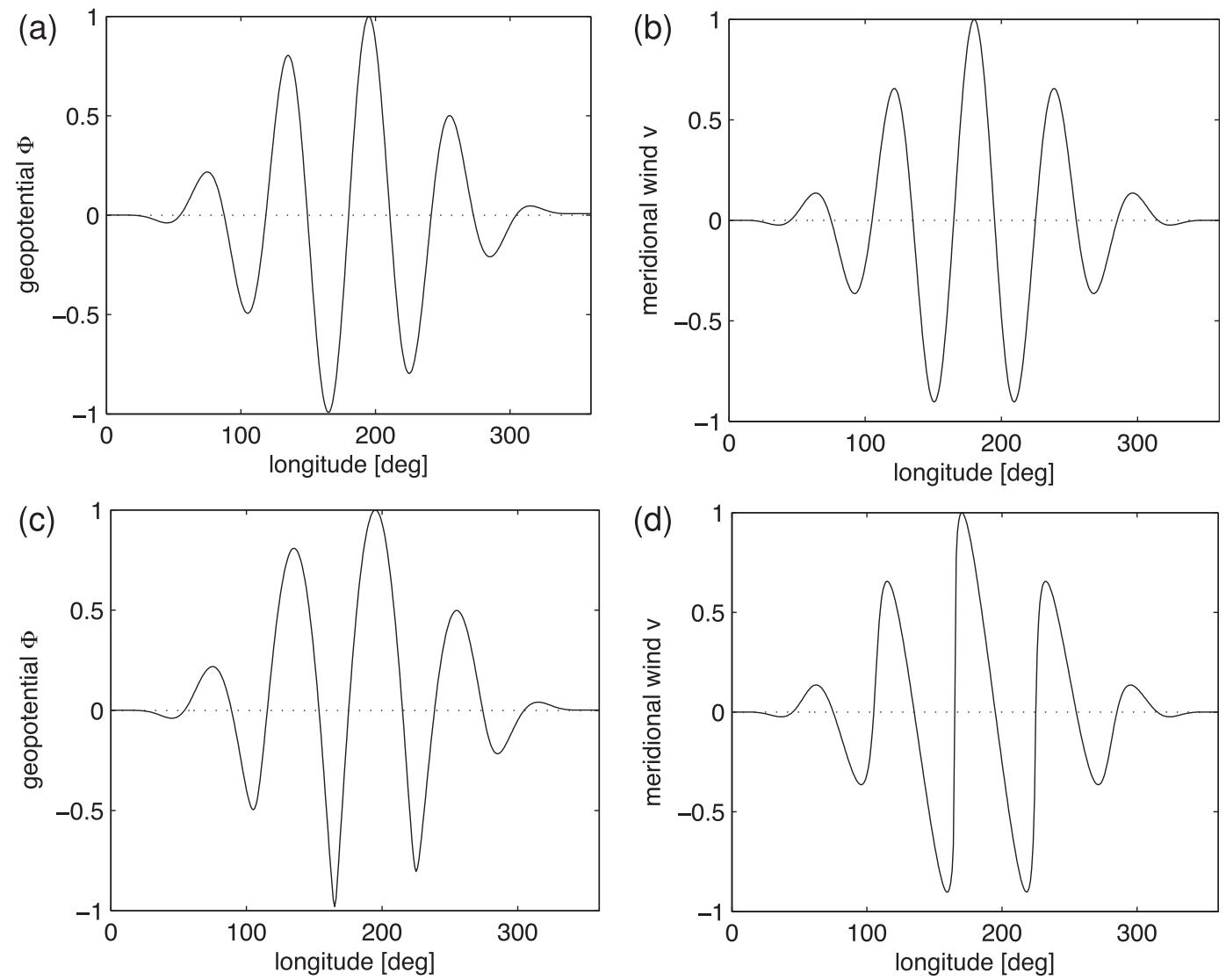

FIG. 1. Two synthetic wave packets: (a),(b) an almost-plane wave packet and (c),(d) a semigeostrophic wave packet. In both cases the wave packet is given either in terms of (left) geopotential or (right) meridional wind. All functions have been normalized such that the maximum value equals 1 .

$$
v(\lambda)=A(\lambda) \cos (s \lambda)
$$

where $s=s_{c}$ is the wavenumber of the carrier wave. However, observed atmospheric Rossby wave packets deviate from the almost-plane wave paradigm to a certain extent. Troughs tend to be narrower than ridges, something well known to the experienced synoptician. This trough-ridge asymmetry is associated with a deviation from quasigeostrophic dynamics (Hakim et al. 2002). The finite-amplitude nature of the Rossby waves and, more generally, the nonlinearity of synoptic-scale dynamics play a role in this context (cf. Wirth 2001). The phenomenon is well captured by semigeostrophic dynamics, as was explicitly pointed out in the seminal work of Hoskins (1975).

In the present paper we show that the deviation from the almost-plane wave paradigm has important implications for Rossby wave packet detection, rendering envelope reconstruction through the Hilbert transform less adequate than suggested by its recent popularity. We start investigating the issue by means of synthetic wave packets (section 2), and we propose remedies to overcome the problem (section 3 ). We then demonstrate that our results are relevant in the real atmosphere by considering specific cases (section 4), which puts into perspective our suggestions to overcome the problems with Rossby wave packet detection. Finally, section 5 summarizes the main results, provides further discussion, and presents our conclusions.

\section{The almost-plane wave paradigm}

In this section we illustrate key aspects by means of synthetic wave packets. In particular we study the differences between almost-plane wave packets and more realistic wave packets. Figure 1 serves for illustration. The connection between the meridional wind $v$ and geopotential $\Phi$ is given by

$$
v=\frac{1}{a f \cos \phi} \frac{\partial \Phi}{\partial \lambda}
$$

where $f$ is the Coriolis parameter, $a$ is the radius of Earth, and $\phi$ denotes latitude. The top row in Fig. 1 
represents an almost-plane wave packet of the form in (2) with $s_{c}=6$ and

$$
A(\lambda)=\sin ^{3}\left(\frac{\lambda}{2}\right)
$$

The bottom row shows a more realistic wave packet (to be defined farther below). Note the symmetry between troughs and ridges in the almost-plane wave packet (Fig. 1a), while troughs are thinner and ridges are broader in the more realistic wave packet (Fig. 1c). Correspondingly, the minima and maxima in $v$ are equidistant in the almost-plane wave packet (Fig. 1b), while for the more realistic wave packet the distance between consecutive minima/maxima is smaller than the distance between consecutive maxima/minima (Fig. 1d). As a consequence, the wave signal in terms of the meridional wind develops some kind of tilt. The distortion of this function from an almost-plane wave packet is qualitatively exactly the same as in the prototypical example from Fig. 1 of Hoskins (1975).

The almost-plane wave packet is shown again in Fig. 2a (thin solid line), together with the corresponding spectrum of zonal wavenumbers (Fig. 2b). In contrast to a pure plane wave, the wave packet contains more than just one wavenumber. However, the distribution of wavenumbers is still restricted to a few wavenumbers and maximizes at the carrier wavenumber $s_{c}$. This is an essential feature of an almost-plane wave packet.

Given $v(\lambda)$, it is the goal of envelope reconstruction to recover $A(\lambda)$ as reliably as possible. We do this here (i) by means of a Hilbert transform and (ii) by means of complex demodulation. For the Hilbert transform method we follow Zimin et al. (2003). The method includes a Fourier transform of the wave signal and, hence, allows the restriction to a specific range of wavenumbers. At this point we do not make use of this latter option (i.e., we use the full range of wavenumbers). For complex demodulation the wave signal $v(\lambda)$ is first multiplied by $\exp \left(i s_{0} \lambda\right)$ with some reference wavenumber $s_{0}$. Thereafter, higher wavenumbers are removed through a Hann filter (i.e., a convolution with a smooth window function) (Press et al. 1996). The final result is obtained by taking twice the absolute value. In our case we choose $s_{0}=s_{c}$, and the full width at half maximum, $\Delta$, of the Hann window is set to be equal to the carrier wavelength (i.e., $\Delta=2 \pi / s_{c}$ ). This makes sure that that the filter practically removes all wavenumbers $s \geq s_{c}$. The need to specify a reference wavenumber and the application of a filter are two major aspects that distinguish complex demodulation from the Hilbert transform technique. Incidentally we note that envelope reconstruction is a nonlinear operation $\mathcal{A}$ working on a function $v$; this means that generally $\mathcal{A}\left(v_{1}+v_{2}\right) \neq \mathcal{A}\left(v_{1}\right)+\mathcal{A}\left(v_{2}\right)$.
As can be seen in Fig. 2a, both methods reconstruct the envelope $A(\lambda)$ very well for the almost-plane wave packet (thick solid and thick dashed lines). When complex demodulation is applied with $s_{0} \neq s_{c}$ (not shown), the quality of the reconstruction decreases. As long as the reference wavenumber $s_{0}$ is close to the carrier wavenumber $s_{c}$, the general shape of $A(\lambda)$ is reconstructed well, but the envelope loses amplitude as the difference between $s_{0}$ and $s_{c}$ increases.

Complex demodulation becomes more problematic when the wave signal is dominated by different wavenumbers in different parts of the domain (Zimin et al. 2003). Consider, for example,

$$
\begin{aligned}
v(\lambda)= & \exp \left[-\left(\lambda-\lambda_{1}\right)^{2}\right] \cos \left(s_{1} \lambda\right) \\
& +\exp \left[-\left(\lambda-\lambda_{2}\right)^{2}\right] \cos \left(s_{2} \lambda\right)
\end{aligned}
$$

with $\lambda_{1}=\pi / 2, \lambda_{2}=3 \pi / 2, s_{1}=4$, and $s_{2}=9$. This function is shown as thin solid line in Fig. 2c. The spectrum of wavenumbers (Fig. 2d) is much broader than in the previous example, featuring two distinct maxima: one at $s=4$ and one at $s=9$. Apparently, the reconstruction by the Hilbert transform works very well (thick solid line in Fig. 2c), and this is partly due to the fact that it includes the entire range of wavenumbers. On the other hand, complex demodulation requires one to specify the reference wavenumber $s_{0}$. Using $s_{0}=4$, the resulting envelope reconstruction (dashed line in Fig. 2c) is poor, and other values for $s_{0}$ do not solve the problem either [see Zimin et al. (2003)].

The better performance of the Hilbert transform technique in the above situation seems to suggest that this technique is superior, and possibly this is the reason for its recent popularity. Yet, the example in (5) is somewhat contrived. In real atmospheric flows there is generally a broad spectrum of zonal wavenumbers, and the reconstruction of the envelope is often not very sensitive to the precise choice of $s_{0}$ (e.g., Chang and Yu 1999).

The point that to our knowledge has not been made so far in this context is the recognition that Rossby wave packets are not almost-plane waves. As mentioned before, the symmetry between troughs and ridges, which exists for a quasigeostrophic Rossby wave, gets lost when going to better approximations of the underlying dynamics. A significant step beyond quasigeostrophy is semigeostrophy, because the latter is able to represent the asymmetry between narrow troughs and wide ridges, which is an important aspect here. The connection between geostrophic and semigeostrophic dynamics is given by the so-called semigeostrophic coordinate 

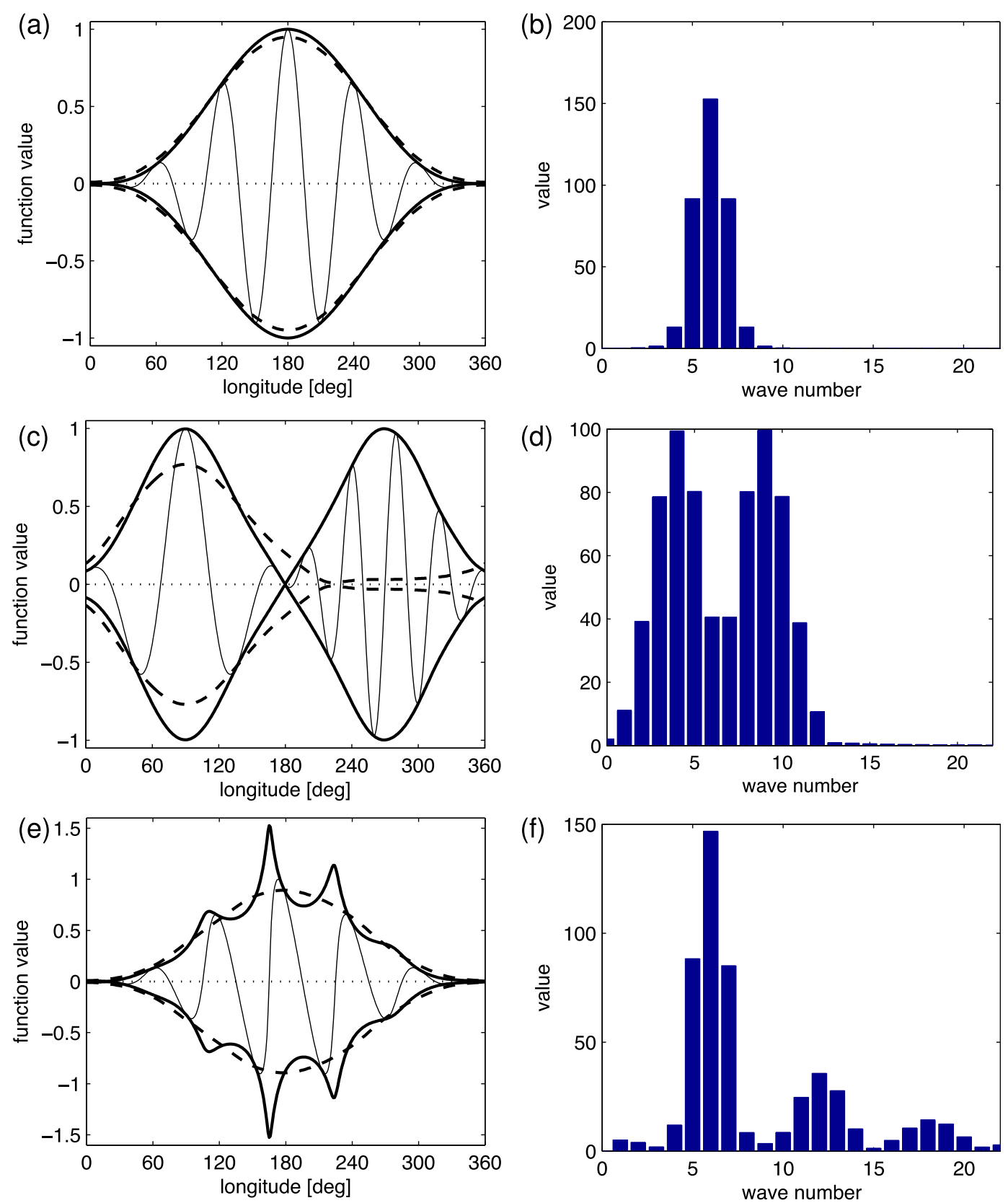

FIG. 2. Three examples of wave packets and their envelope reconstruction using two different methods. (left) The meridional wind $v(\lambda)$ (thin solid) together with the envelope reconstruction-depicted as $\pm A(\lambda)$-through the Hilbert transform (thick solid) and through complex demodulation (thick dashed); the thin dotted line is the zero line. (right) The corresponding zonal wavenumber spectrum of $v(\lambda)$. (a),(b) The almost-plane wave packet; (c),(d) two spatially separate wave packets; and (e),(f) a more realistic wave packet with an asymmetry between troughs and ridges resulting from a semigeostrophic coordinate transformation.

transformation (Hoskins 1975). The essence of the latter can be obtained by applying the following transformation:

$$
\hat{\lambda}=\lambda-\alpha v,
$$

to the original wave packet from Fig. 2a (with $\alpha$ being a positive constant). The dependence of $\hat{\lambda}$ on $\lambda$ is monotonic and, hence, the transformation is regular as long as $\alpha$ is small enough, which is satisfied for our current choice $\alpha=0.12$. The new wave packet is defined in transformed coordinates as

$$
\hat{v}(\hat{\lambda})=A(\hat{\lambda}) \cos (s \hat{\lambda})
$$



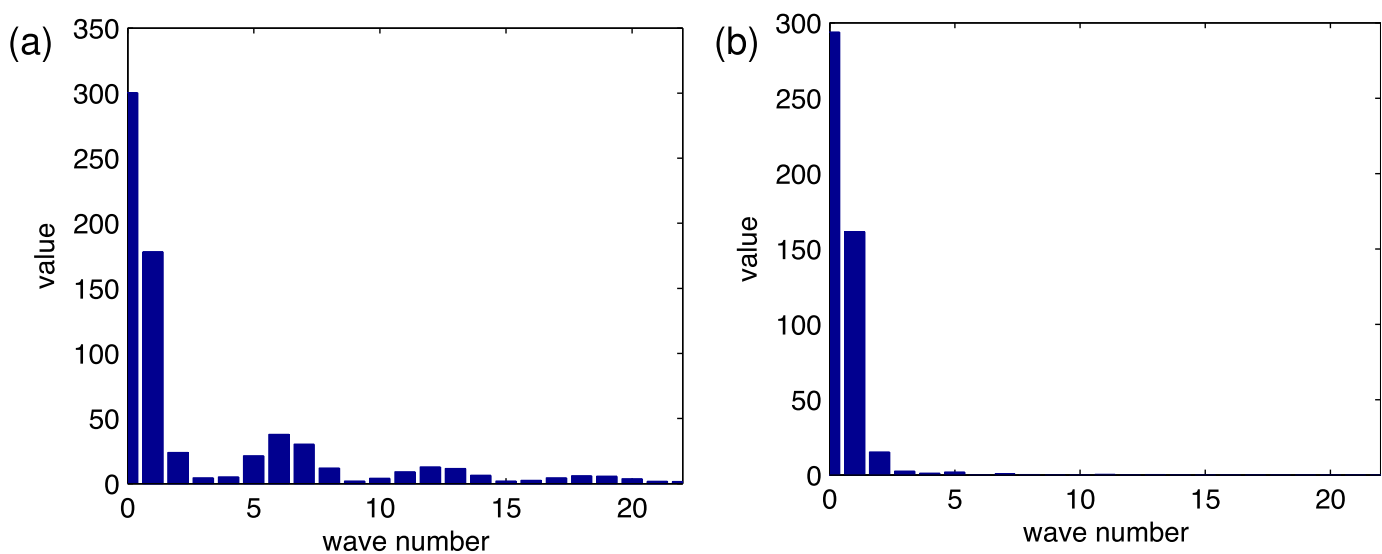

FIG. 3. Wavenumber spectra of the reconstructed envelopes shown in Fig. 2e: (a) using the Hilbert transform and (b) using complex demodulation.

with amplitude function $A$ as given in (4). Transforming back to physical space (i.e., to coordinate $\lambda$ ), the new wave packet $\hat{v}(\lambda)$ is shown in Fig. 2e (thin solid). Figure $2 \mathrm{e}$ also shows the envelope reconstructions through the Hilbert transform (thick solid) and through complex demodulation with $s_{0}=6$ (thick dashed). Now complex demodulation performs better than the Hilbert transform, with the latter showing strong wiggles on the scale of the dominant wavenumber. Note, however, that the excellent performance of complex demodulation is partly due to the fact that we exploited the knowledge of $s_{c}$ in order to specify the reference wavenumber $s_{0}$ appropriately; obviously, this may not be so straightforward in real world cases.

To elucidate the poor performance of the Hilbert transform reconstruction, we consider the wavenumber spectrum of the wave packet $v(\lambda)$ (Fig. 2f). The low wavenumber peak is very similar as in the first example (cf. Figs. $2 \mathrm{f}$ and $2 \mathrm{~b}$ ), but in addition there is a series of peaks at higher harmonics $\left(s=n s_{c}\right.$ with $\left.n=2,3, \ldots\right)$. The latter characterizes the deviation from an almostplane wave packet. By design, the complex demodulation technique is able to effectively remove these higher harmonics, because it implies smoothing through a spatial filter. On the other hand, lack of smoothing renders the Hilbert transform more vulnerable and, hence, more deficient regarding this effect.

The existence of significant higher harmonics in the reconstructed Hilbert envelope can become a serious problem when trying to identify wave packet objects. Recently, wave packet objects have been defined as those parts of the envelope that exceed a user-defined threshold (Glatt et al. 2011; Glatt and Wirth 2014). As a result, the wiggles from higher harmonics may lead to the disintegration of the object: what should be considered as one wave packet actually splits into several (smaller) subpackets [e.g., Fig. 5 in Glatt and Wirth (2014)] owing to the so-called camel effect that occurs when there are two relative maxima in close proximity to each other (Wernli et al. 2008).

\section{How to get around the problem?}

As we have seen, the higher harmonics of $v(\lambda)$ may prevent a reliable envelope reconstruction through the Hilbert transform. The point is made more explicit in Fig. 3, where we show the wavenumber spectra of the reconstructed envelopes. By construction, wavenumbers $s \geq s_{c}$ (with $s_{c}=6$ in the present example) are practically absent in the reconstruction from complex demodulation (Fig. 3b); this is due to the smoothing applied in this technique. On the other hand, the Hilbert transform does not imply any smoothing; therefore, it is not surprising that the reconstructed envelope (Fig. 3a) contains the carrier wavenumber $s_{c}$ and its harmonics, resembling the higher harmonics from the original wave packet (see Fig. 2f).

A straightforward improvement of the Hilbert transform reconstruction is, therefore, to remove these higher frequencies by suitably filtering the envelope. One possibility consists in the restriction to a specific range of wavenumbers, which can easily be implemented and that was suggested by Zimin et al. (2003) as part of their algorithm. Restriction to $4 \leq s \leq 15$ [as used by Glatt and Wirth (2014) in their climatology] reduces the wiggles of the envelope only marginally (Fig. 4, thick dashed). On the other hand, restriction to $4 \leq s \leq 9$ [as used in Zimin et al. (2003)] removes the wiggles altogether (Fig. 4, thin solid). The latter becomes plausible upon inspection of the spectrum of the original wave packet in Fig. 2f: removal of $s>9$ removes all higher 


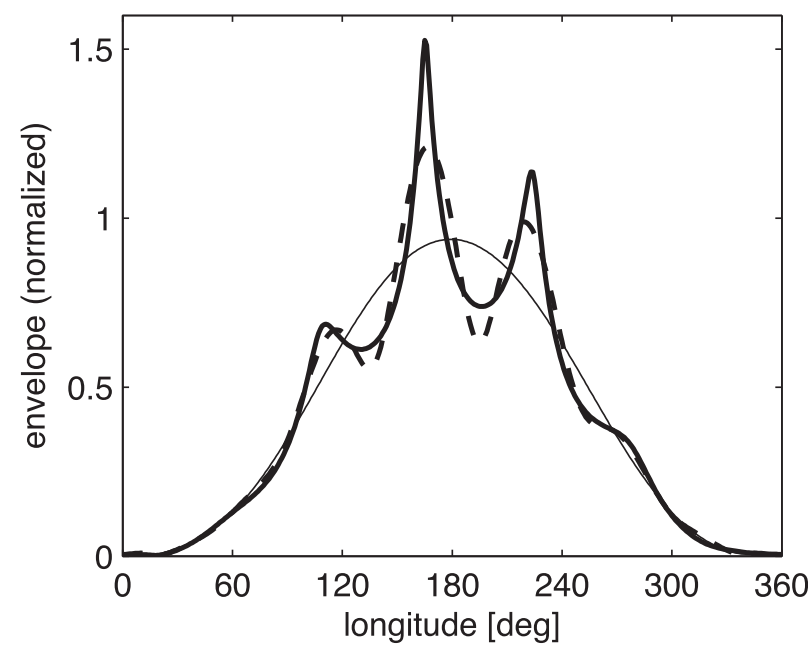

FIG. 4. Envelope reconstructions for the wave packet from Fig. 2e resulting from the Hilbert transform method with different restrictions to zonal wavenumbers: no restriction in wavenumbers (thick solid), wavenumbers restricted to $4 \leq s \leq 15$ (thick dashed), and wavenumbers restricted to $4 \leq s \leq 9$ (thin solid).

harmonics from the wave signal thus turning it effectively into an almost-plane wave packet (cf. Fig. 2b).

Another way of filtering is the convolution of the original wave signal with a suitably chosen window function (Press et al. 1996). Obviously this is equivalent to some manipulation in Fourier space, but it is arguably preferable to the selection of a finite range of wavenumbers, because it corresponds to a more gradual transition from wavenumbers that are accounted for to wavenumbers that are discarded. Here, we choose a Hann filter with a full width at half maximum of $\Delta=$ $2 \pi / s_{c}$. The resulting smoothed envelope is depicted by the thick dashed line in Fig. 5a. By design, the wiggles on the extracted envelope from Fig. 2e (thick solid line) have been removed.

Unfortunately, one side effect of filtering or smoothing is the loss of variance of the reconstructed envelope. In particular this can reduce the maximum amplitude. In our example the maximum amplitude of the true envelope should be 1 according to (4), but in fact the maxima of the reconstructed envelopes in Fig. 4 (thin solid) and in Fig. 5a (thick dashed) are less than 1. In addition to decreasing the relative maxima, filtering generally increases relative minima, while the average value is unaffected. In other words, filtering reduces the relative difference between the minima and the maxima of the envelope. In the context of defining wave packet objects this may be quite detrimental, because related algorithms often apply a user-specified threshold in the sense that only those parts of the envelope contribute to the object that exceed the threshold. Loss of variance in the reconstructed envelope means that the object identification becomes more sensitive to the precise value of the threshold, which is undesirable. For this reason we propose that, in addition to filtering, the algorithm should include some "variance recovery." This is implemented by inflating the deviation from the mean by a factor that guarantees that the variance of the original envelope is recovered; thereafter, the function is set to zero in those areas where variance recovery would produce negative values-simply because the envelope must be a nonnegative function. The resulting envelope is shown as bold solid line in Fig. 5a. Apparently, variance recovery works very well in our example.

We now propose another method to deal with the wiggles on the Hilbert reconstruction of the envelope. This solution has not been mentioned in the literature
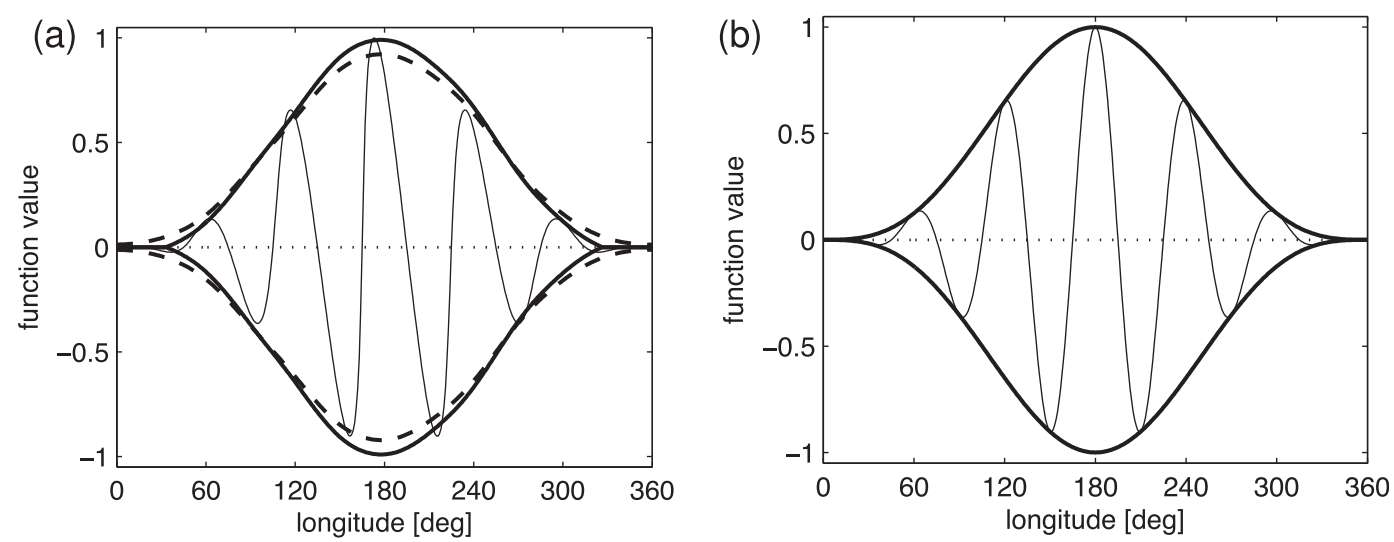

FIG. 5. Improved envelope reconstructions-again depicted as $\pm A(\lambda)$-based on the Hilbert transform technique. (a) The original wave packet (thin solid) together with the reconstructed Hilbert envelope after smoothing (thick dashed) and after smoothing with variance recovery (thick solid). (b) The wave packet (thin solid) after the back transformation in (8) was applied to the original wave packet, together with the envelope reconstruction (thick solid) using a simple Hilbert transformation on the transformed wave packet. 
before, but it appears to be straightforward after what we have shown above. By design the higher harmonics in our synthetic wave packet were introduced by the transformation in (6). One, thus, should be able to get rid of them by applying the corresponding back transformation,

$$
\hat{\lambda}=\lambda+\alpha v
$$

to the wave packet. Figure 5 shows that this is, indeed, the case-by necessity in this synthetic example. Note that this approach does not imply any smoothing.

\section{Examples with observed wave packets}

While the basic ideas from the previous section appear quite straightforward, it is not so clear to what extent they work for atmospheric Rossby wave packets. In particular, real wave packets are characterized by a broad spectrum of wavenumbers and it is not easy to define a carrier wavenumber $s_{c}$, which is needed for complex demodulation and for the application of a filter. In addition, it is not clear to what extent the semigeostrophic transformation is able to undo the troughridge asymmetry in the context of a Rossby wave packet.

In this section we shall investigate these issues in the framework of observed cases. As data we use the meridional wind $v$ at $300 \mathrm{hPa}$ from Interim European Centre for Medium-Range Weather Forecasts (ECMWF) ReAnalysis (ERA-Interim; Dee et al. 2011) on a latitudelongitude grid with $1.5^{\circ}$ spatial resolution. Obviously, the meridional wind $v(\lambda, \phi)$ and its envelope $A(\lambda, \phi)$ are now functions of both longitude and latitude. As before, we proceed to reconstruct the envelope $A(\lambda, \phi)$ separately for each latitude bin, except that we now restrict the zonal wavenumbers to the interval $4 \leq s \leq 15$ [following Glatt and Wirth (2014)].

A key aspect for some of our reconstruction methods is the application of a filter (which in our case is a Hann filter), and this requires the determination of the filter width. Generally, the wavenumber spectrum of $v(\lambda)$ has a peak somewhere in the synoptic scales, but it typically extends over a large range of wavenumbers. Simply using the wavenumber $s_{\max }$ at which the spectrum maximizes would imply substantial jumps from one latitude bin to the next. The latter is clearly unphysical, because the filter width $\Delta$ is meant to apply to the entire wave packet; it should, therefore, vary smoothly with latitude. For this reason we still diagnose $s_{\max }$ at each latitude bin, but thereafter apply a filter in latitude such that one obtains a gradual variation of $s_{\max }$ with latitude. We found that a moving average over $20^{\circ}$ of latitude performs well in the present context. In our examples, the resulting "dominant wavenumber" $s_{d}(\phi)$ varies between $s_{d}=3$ and $s_{d}=10$, with lower values at higher latitudes. The filter width for our Hann window is set to $\Delta=\pi / s_{d}$, which turned out to be satisfying in all real cases that we considered. Note that this value of $\Delta$ is smaller than what we used in our synthetic examples in section 3 ; this is because we wanted to apply as little smoothing as possible for both the complex demodulation and the Hilbert transform technique.

The semigeostrophic coordinates $\left(\lambda_{\mathrm{sg}}, \phi_{\mathrm{sg}}\right)$ in spherical geometry are defined by

$$
\begin{aligned}
& \lambda_{\mathrm{sg}}=\lambda+\frac{v_{g}}{f a \cos \phi}, \\
& \phi_{\mathrm{sg}}=\phi-\frac{u_{g}}{f a},
\end{aligned}
$$

where angles are given in radians, $f=2 \Omega \sin \phi$ is the Coriolis parameter, $\Omega$ is the angular velocity of Earth's rotation, and the wind $\left(u_{g}, v_{g}\right)$ is obtained from geopotential $\Phi$ through

$$
\begin{aligned}
& u_{g}=-\frac{1}{f a} \frac{\partial(\Phi-\bar{\Phi})}{\partial \phi} \\
& v_{g}=\frac{1}{f a \cos \phi} \frac{\partial \Phi}{\partial \lambda}
\end{aligned}
$$

In (11) we subtracted the zonal mean geopotential $\bar{\Phi}$, because the latter would only lead to a constant shift of latitude according to (10), which is inconsequential in our application. The transformation is regular as long as the vertical component of absolute geostrophic vorticity is positive (Hoskins 1975). In the present application, the transformation from the transformed grid back to the regular latitude-longitude grid is done by linear interpolation of the nearest four grid points (details can be found in the appendix). This method effectively regularizes the transformation.

We consider a specific case of a Rossby wave packet that occurred on the Northern Hemisphere during the first half of August 2002. This wave packet was investigated previously in some detail (e.g., Enomoto et al. 2007; Glatt and Wirth 2014), presumably because it was associated with heavy rainfall and catastrophic flooding in parts of central Europe. Figure 6a presents a conventional Hovmoeller diagram showing clear signs of downstream development (Chang 1993).

Figure 7 compares four different methods of wave packet reconstruction for the above wave packet on 7 August 2002. On that date, there is a wave signal over North America and the western part of the North Atlantic Ocean. It is quite apparent from the previous 


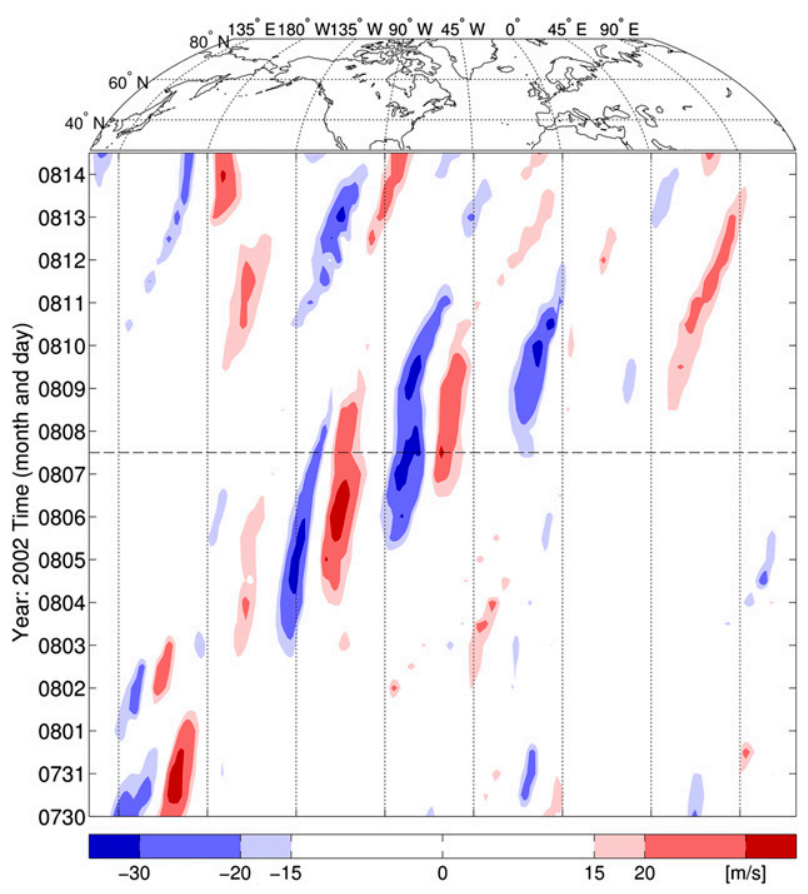

FIG. 6. Hovmoeller diagram of the meridional wind $v\left(\mathrm{~m} \mathrm{~s}^{-1}\right)$ at $300 \mathrm{hPa}$ for an episode in August 2002. The data were averaged between $40^{\circ}$ and $60^{\circ} \mathrm{N}$. The horizontal dashed line indicates the time of the snapshots in Fig. 7.

figure that this wave signal should be considered as one single wave packet-any split must be considered as spurious. Figure $7 \mathrm{a}$ shows the result using the Hilbert transform. The southerly and northerly wind maxima (red and blue contours) occur in pairs, with larger spacing in between (this feature is also apparent in the Hovmoeller diagram in Fig. 6). The existence of such pairs exactly corresponds to what one expects from semigeostrophic dynamics, namely, that troughs are thinner than ridges. As a consequence, the algorithm tends to produce an envelope with two distinct relative maxima, which are separated by a certain distance in longitude. For the threshold chosen here to define a wave packet object (thick black contour), the wave packet fragments into two parts.

Smoothing the reconstructed envelope through a Hann filter (including variance recovery) greatly reduces the problem (Fig. 7b). The modified algorithm detects one single Rossby wave packet. Similarly, complex demodulation successfully reconstructs one single Rossby wave packet (Fig. 7c). As pointed out before, this is not surprising, because complex demodulation implicitly includes smoothing in the longitudinal direction.

Finally, Fig. 7d shows the envelope reconstruction for which the Hilbert transform was applied to the wind field after semigeostrophic coordinate transformation. The wind field $v$ in Fig. 7d differs from the wind field in all previous panels; most notably, the tendency for negative and positive extrema in $v$ to be organized into distinct pairs has nearly vanished. In other words, the distance between successive extrema of $v$ is approximately equal, in distinct contrast to the previous three panels. As a consequence, the Hilbert transform has no problem in detecting this wave feature as one single wave packet, even without smoothing or filtering. We conclude that in the present case the predictions from our earlier synthetic examples hold true: the specific distortions inherent in semigeostrophic dynamics produce a tendency for wave packet fragmentation, which can be avoided to a large extent by doing the envelope reconstruction in semigeostrophic coordinate space rather than in physical space.

Considering the different panels in Fig. 7 we admit that the choice of the threshold to define the wave packet object (bold black contour in all panels) is arbitrary, and modifying the threshold may split one wave packet into two or merge two wave packets into one. This is always the case as long as one has to cope with the camel effect (Wernli et al. 2008). To be sure, the camel effect is not always completely eliminated through our proposed remedies. However, the strength of the camel effect is reduced, and this reduces the likelihood of spurious splits or mergers. In other words, the results become less sensitive to the choice of the threshold, which is a desirable feature.

We programmed a web page on which we posted daily maps with our diagnostics, and we studied these maps for several months. In many cases, the results were perfectly consistent with the behavior described above. However, we also found cases in which the Hilbert transform applied in semigeostrophic space did not do a good job, but rather led to an enhanced tendency for wave packet fragmentation. Figure 8 provides an example, for which, again, the corresponding Hovmoeller diagram (not shown) indicates that the wave signal should be considered as one single wave packet. While in this case the original Hilbert transform method (Fig. 8a) performs well in finding a single wave packet, the reconstruction in semigeostrophic space (Fig. 8b) fails and the wave packet fragments into two parts. The difficulty in this case (and similar other cases) seems to arise from a large quasi-stationary trough off the east coast of North America. The wave packet of interest travels around this trough, which means that the trough structure should be considered as part of the background flow rather than part of the wave packet. Going from physical to semigeostrophic space makes this quasi-stationary trough even broader and leads (in this case) to the fragmentation of the wave packet.

To cope with such a situation, we propose to only use the synoptic- and smaller-scale part of the wind field for 
(a)

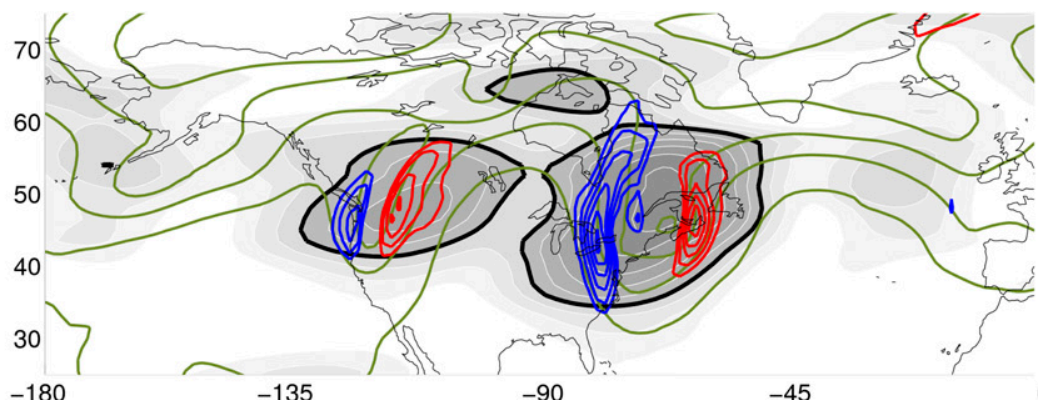

(b)

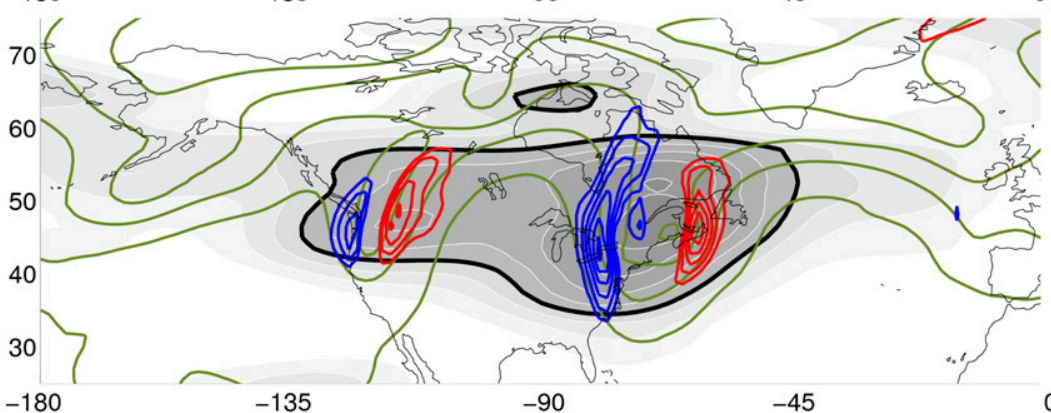

(c)

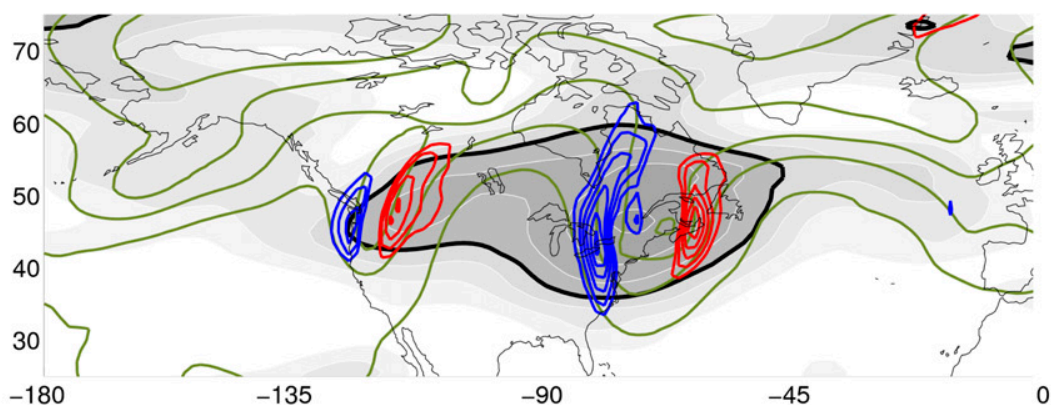

(d)

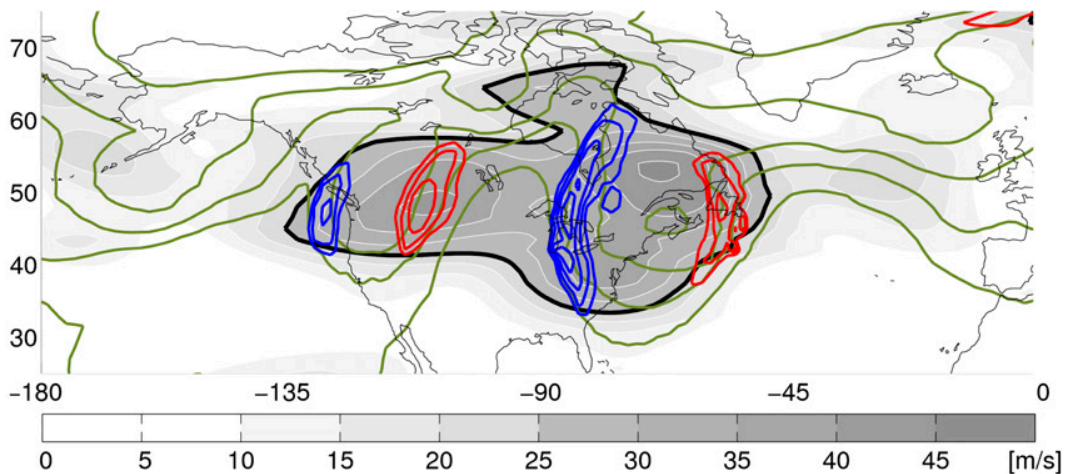

FIG. 7. Envelope reconstruction for a wave packet observed over North America at 1200 UTC 7 Aug 2002. Green contours depict the 300-hPa geopotential (contours every $0.15 \times$ $10^{4} \mathrm{~m}^{2} \mathrm{~s}^{-2}$ between 8.85 and $9.45 \times 10^{4} \mathrm{~m}^{2} \mathrm{~s}^{-2}$ ). Red and blue contours represent the $300-\mathrm{hPa}$ meridional wind $v\left(\mathrm{~m} \mathrm{~s}^{-1}\right.$; negative contours $-30,-35, \ldots$ in blue; positive contours $+30,+35$, $\ldots$ in red). The shading depicts the envelope $A(\lambda, \phi)$; the thick black contour corresponds to the $25 \mathrm{~m} \mathrm{~s}^{-1}$ contour of $A(\lambda, \phi)$, which can be interpreted as the bounding contour of a wave packet object. (a) Original envelope reconstruction using the Hilbert transform with zonal wavenumbers restricted to $4 \leq s \leq 15$. (b) As in (a), but with additional smoothing through a Hann filter followed by variance recovery. (c) Envelope reconstruction using complex demodulation. (d) Envelope reconstruction using the Hilbert transform in semigeostrophic coordinate space. 
(a)

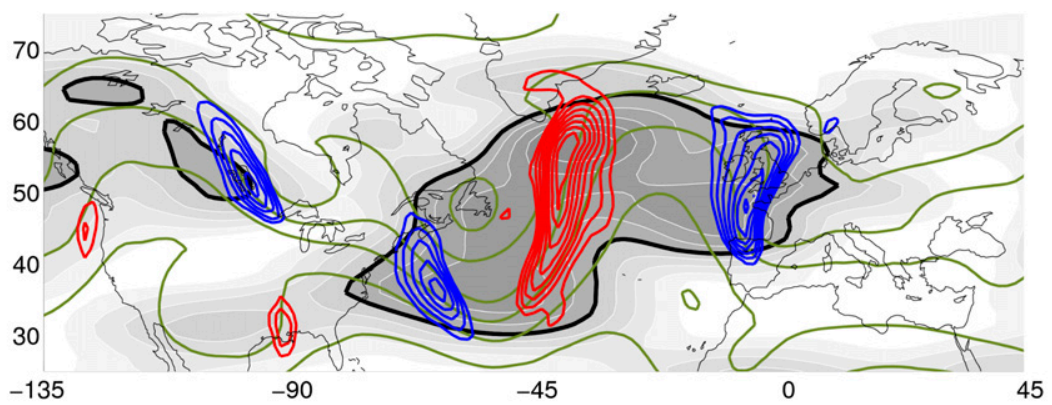

(b)

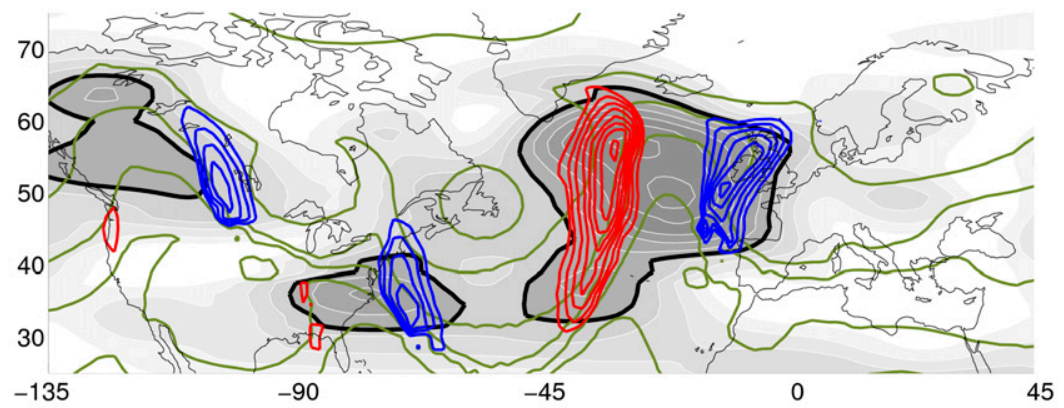

(c)

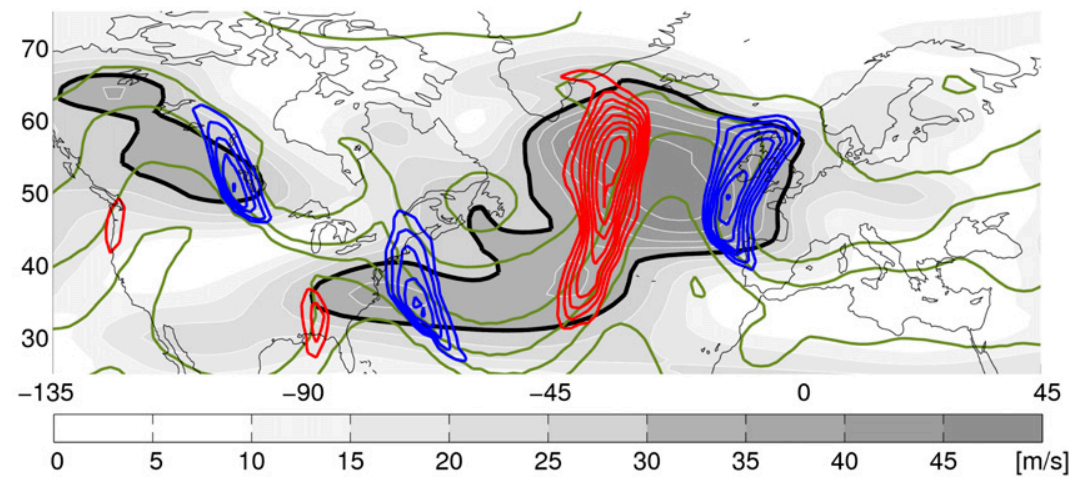

FIG. 8. Envelope reconstruction for a wave packet observed over the North Atlantic Ocean at 0000 UTC 18 Jan 2004. Plot conventions are as in Fig. 7, except here the thick black contour corresponds to $30 \mathrm{~m} \mathrm{~s}^{-1}$ and the contours for the geopotential are drawn every $0.2 \times 10^{4} \mathrm{~m}^{2} \mathrm{~s}^{-2}$ between 8.1 and $9.3 \times 10^{4} \mathrm{~m}^{2} \mathrm{~s}^{-2}$. (a) Original envelope reconstruction using the Hilbert transform with zonal wavenumbers restricted to $4 \leq s \leq 15$. (b) Envelope reconstruction using the Hilbert transform in semigeostrophic coordinates. (c) Envelope reconstruction using the Hilbert transform in modified semigeostrophic coordinates (see text).

the semigeostrophic coordinate transformation. The rationale for this approach is that the quasi-stationary planetary waves can be considered as part of the background flow, which should not be included into the coordinate transformation. More specifically we use $u_{g}\left(\left[s_{1}, s_{2}\right]\right)$ and $v_{g}\left(\left[s_{1}, s_{2}\right]\right)$ instead of $u_{g}$ and $v_{g}$ in the semigeostrophic coordinate transformation in (9) and (10), where the notation $\left(\left[s_{1}, s_{2}\right]\right)$ indicates the restriction to zonal wavenumbers $s$ satisfying $s_{1} \leq s \leq s_{2}$. Moreover, this transformation is applied to $v\left(\left[s_{1}, s_{2}\right]\right)$ rather than to the full wind field $v$. In the current example we use $\left[s_{1}, s_{2}\right]=[4,17]$, that is, a slightly higher upper limit for the range of zonal wavenumbers than before, because the transformation broadens the troughs and effectively reduces the range of zonal wavenumbers. For the ensuing Hilbert transformation we use, again, $\left[s_{1}, s_{2}\right]=[4,15]$. Figure $8 \mathrm{c}$ shows the result. As desired, the tendency for wave packet fragmentation (as in Fig. 8b) is now largely absent; instead the algorithm reconstructs a single wave packet, even though its amplitude in the western half is reduced in comparison with the original reconstruction from Fig. 8a. The latter is due to the fact that the new algorithm interprets the strong trough off the east coast of North America at least partly as background flow, which is not part of the wave packet.

How does the modified semigeostrophic algorithm work in straightforward cases for which the unmodified semigeostrophic algorithm works well? To address this 


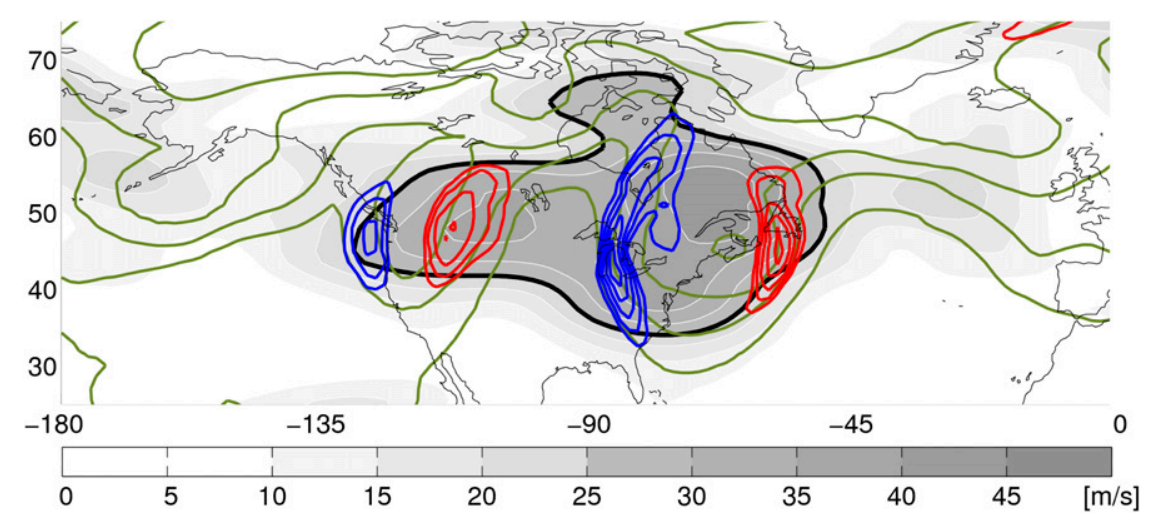

FIG. 9. Envelope reconstruction for the wave packet from Fig. 7 using the Hilbert transform method in modified semigeostrophic coordinate space. Plot conventions are as in Fig. 7.

question, we applied the modified algorithm to the wave packet from Fig. 7. The result is given in Fig. 9. Comparison with Fig. 7d shows that in this case the modification leaves the wave packet reconstruction practically unchanged, as desired.

\section{Summary, discussion, and conclusions}

Envelopes of Rossby wave packets have previously been diagnosed from the meridional wind $v$ using either complex demodulation or the Hilbert transform. The latter appears preferable, because, unlike complex demodulation, it does not require specification of a reference wavenumber (which is not known a priori) and it does, by itself, not imply any smoothing.

However, the Hilbert transform suffers from a fundamental deficiency that has not been pointed out so far: it is fraught with a tendency to split a single Rossby wave packet into several fragments. Through the use of synthetic wave packets we have shown that this problem arises when the wave signal deviates from the almostplane wave paradigm in the sense predicted by semigeostrophic theory. In this case the fundamental frequency from the wave signal as well as its higher harmonics are incorporated into the reconstructed envelope, leading to significant wiggles. On the other hand, complex demodulation does not suffer from this problem, because the implied smoothing removes those undesired frequencies.

We demonstrated that this phenomenon is real and produces a tendency toward wave packet fragmentation when applying the Hilbert transform to observed cases. As expected from semigeostrophic theory, this is associated with troughs being narrower than ridges such that extrema in $v$ are not evenly spaced in longitude.

A possible solution is to resort to complex demodulation, since this technique involves smoothing on the scale of the dominant wavenumber. Another solution is to combine the Hilbert transform with some modest smoothing by convolution with (as we suggest) a Hann filter in physical space. This significantly reduces the problem without the additional need to specify a unique reference wavenumber. The latter is roughly equivalent to the suggestion of Zimin et al. (2003) who combined the Hilbert transform with a filter in spectral space by limiting the zonal wavenumbers to a finite range $\left[s_{\min }, s_{\max }\right]$ covering the synoptic scales. Our analysis reveals the reason why such a filter is necessary (i.e., it uncovers the underlying problem for which smoothing or filtering is the solution).

Whether the filtering is done in physical or in Fourier space, in both cases one is left with the (somewhat undesirable) fact that smoothing requires the choice of a filter shape and width and reduces the variance of the reconstructed envelope. In the case of the Hilbert transform technique, the loss of variance can be counteracted to a large extent by some form of variance recovery. The latter is recommended in our present context, because it ensures that the definition of wave packet objects based on envelope thresholds does not become overly sensitive to the choice of the threshold. We also suggested an algorithm for selecting the filter width, which appears to work reliably in observed cases.

Yet another method to reduce the problem with the Hilbert transformation is the application of a semigeostrophic coordinate transformation. This effectively reduces semigeostrophic dynamics to quasigeostrophic dynamics and presumably removes the underlying problem. In the framework of an observed case we have demonstrated that the technique reduces the tendency of spurious wave packet fragmentation, and this is consistent with our experience from a large number of cases. However, unfortunately in some cases the semigeostrophic coordinate transformation works in the opposite direction 
(i.e., it spuriously increases the tendency of wave packet fragmentation). Such problematic cases seem to be characterized by the wave packet propagating along a low wavenumber quasi-stationary background flow. We, therefore, suggested to exclude the lowest wavenumbers from the semigeostrophic coordinate transformation. This modification improved the situation in all cases that we considered.

Although both smoothing and the semigeostrophic coordinate transformation work well in practice, we find the latter solution conceptually more satisfying. This is because the semigeostrophic coordinate transformation does not imply the loss of physical information and is well motivated by the underlying dynamics.

A recent paper by Donohoe and Battisti (2009) has shown that the amplitude asymmetry between surface cyclones and anticyclones depends quite sensitively on the method of filtering previously applied to the raw data, with implications for feature tracking. At first sight this seems to suggest that the filtering method might affect the troughridge asymmetry and the resulting envelope reconstruction in our case as well. However, we are concerned here not so much with the difference in amplitude between troughs and ridges, but rather with their spatial extent (i.e., narrow troughs vs wide ridges). Concerning the asymmetry in spatial extent it turns out (no figure shown) that temporal instead of spatial filtering does not remove the difference between narrow ridges and wide troughs. The application of a semigeostrophic coordinate transformation is beneficial in both cases (i.e., no matter whether the data were previously filtered spatially or temporally).

Zimin et al. (2006) improved their own method of envelope reconstruction by performing the Fourier transform along streamlines of the background flow instead along circles of constant latitude. Although this method requires additional choices to be made and is computationally much more expensive, it does lead to an improvement in some cases, especially when the direction of wave packet propagation has a substantial meridional component. On the other hand, it does generally not eliminate the problem that we are concerned with in the present paper: wave packets may still get fragmented as a result of the trough-ridge asymmetry, because the latter is independent of the direction of wave propagation.

What have we learned in the end? To be sure, the definition and detection of Rossby wave packets is a thorny issue, because there is no "truth" with which to compare. Different techniques yield different results, which renders the whole concept of a Rossby wave packet somewhat elusive (Glatt et al. 2011) —even though not completely useless. A key problem is to determine whether a wave signal is a single wave packet or rather a succession of two or more separate wave packets.
Often this decision is difficult to make, and objective algorithms do not help either, because in these the decision is made implicitly by the choice of some parameters [such as a threshold value, see e.g., Glatt and Wirth (2014)]. To the extent that an algorithm implies substantial smoothing this leads to the merger of independent wave packets, which is undesirable. A partial solution would be to consider a combination of methods [as implicit in Glatt et al. (2011)], and indeed this has recently been used by Souders et al. (2014) in order to evaluate a new automated method for tracking Rossby wave packets. However, one point became very clear from the present work: the fragmentation of wave packets resulting from their semigeostrophic nature should be avoided in any case, because it is a simple consequence of the underlying dynamics and has nothing to do with the integrity of the wave packet. Possibly the most satisfying method to avoid this problem without any smoothing is the use of a Hilbert transform after a modified semigeostrophic coordinate transformation as presented in this paper.

Acknowledgments. The authors thank Brian Hoskins for suggesting the use of the semigeostrophic coordinate transformation, as well as Ilona Glatt and Jachim Eichhorn for helpful discussions. Three anonymous reviewers provided numerous insightful comments, which led to a significant improvement of the presentation. This work was partly funded by DFG Grant WI-1685/8-2.

\section{APPENDIX}

\section{Semigeostrophic Coordinate Transformation}

The semigeostrophic coordinate transformation yields the wind $v$ on the transformed grid $\left(\lambda_{\mathrm{sg}}, \phi_{\mathrm{sg}}\right)$. This needs to be interpolated back onto the regular longitudelatitude grid $(\lambda, \phi)$. For each grid point $\left(\lambda_{i}, \phi_{i}\right)$ of the latter grid, we consider the five nearest grid points of the transformed grid. The distance $\Delta_{i, k}$ between these five transformed grid points $\left(\lambda_{\mathrm{sg}, k}, \phi_{\mathrm{sg}, k}\right), k=1,2, \ldots, 5$, and the grid point $\left(\lambda_{i}, \phi_{i}\right)$ is

$$
\Delta_{i, k}=\sqrt{\cos ^{2} \phi_{i}\left(\lambda_{i}-\lambda_{\mathrm{sg}, k}\right)^{2}+\left(\phi_{i}-\phi_{\mathrm{sg}, k}\right)^{2}} .
$$

The value of $v$ on the grid point $\left(\lambda_{i}, \phi_{i}\right)$ is then computed as

$$
v\left(\lambda_{i}, \phi_{i}\right)=\frac{\sum_{k=1}^{4} v\left(\lambda_{\mathrm{sg}, k}, \phi_{\mathrm{sg}, k}\right)\left(\Delta_{5, i}-\Delta_{k, i}\right)}{\sum_{k=1}^{4}\left(\Delta_{5, i}-\Delta_{k, i}\right)},
$$

where $\Delta_{5, i}=\max _{k} \Delta_{k, i}$ 


\section{REFERENCES}

Chang, E. K. M., 1993: Downstream development of baroclinic waves as inferred from regression analysis. J. Atmos. Sci., 50, 2038-2053, doi:10.1175/1520-0469(1993)050<2038: DDOBWA $>2.0 . \mathrm{CO} ; 2$.

1999: Characteristics of wave packets in the upper troposphere. Part II: Seasonal and hemispheric variations. J. Atmos. Sci., 56, 1729-1747, doi:10.1175/1520-0469(1999)056<1729: COWPIT $>2.0 . \mathrm{CO} ; 2$

- 2005: The impact of wave packets propagating across Asia on Pacific cyclone development. Mon. Wea. Rev., 133, 1998-2015, doi:10.1175/MWR2953.1.

—, and I. Orlanski, 1993: On the dynamics of a storm track. J. Atmos. Sci., 50, 999-1015, doi:10.1175/1520-0469(1993)050<0999: OTDOAS $>2.0 . \mathrm{CO} ; 2$.

—, and D. B. Yu, 1999: Characteristics of wave packets in the upper troposphere. Part I: Northern Hemisphere winter. J. Atmos. Sci, 56, 1708-1728, doi:10.1175/1520-0469(1999)056<1708: COWPIT $>2.0 . \mathrm{CO} ; 2$.

Dee, D. P., and Coauthors, 2011: The ERA-interim reanalysis: Configuration and performance of the data assimilation system. Quart. J. Roy. Meteor. Soc., 137, 553-597, doi:10.1002/ qj.828.

Donohoe, A., and D. S. Battisti, 2009: The amplitude asymmetry between synoptic cyclones and anticyclones: Implications for filtering methods in feature tracking. Mon. Wea. Rev., 137, 3874-3887, doi:10.1175/2009MWR2837.1.

Enomoto, T., W. Ohfuchi, H. Nakamura, and M. A. Shapiro, 2007: Remote effects of tropical storm Cristobal upon a cut-off cyclone over Europe in August 2002. Meteor. Atmos. Phys., 96, 29-42, doi:10.1007/s00703-006-0219-2.

Glatt, I., and V. Wirth, 2014: Identifying Rossby wave trains and quantifying their properties. Quart. J. Roy. Meteor. Soc., 140, 384-396, doi:10.1002/qj.2139.

— A. Dörnbrack, S. Jones, J. Keller, O. Martius, A. Müller, D. H. W. Peters, and V. Wirth, 2011: Utility of Hovmöller diagrams to diagnose Rossby wave trains. Tellus, 63A, 9911006, doi:10.1111/j.1600-0870.2011.00541.x.

Hakim, G. J., C. Snyder, and D. J. Muraki, 2002: A new surface model for cyclone-anticyclone asymmetry. J. Atmos. Sci., 59, 2405-2420, doi:10.1175/1520-0469(2002)059<2405: ANSMFC $>2.0 . \mathrm{CO} ; 2$
Hoskins, B. J., 1975: The geostrophic momentum approximation and the semi-geostrophic equations. J. Atmos. Sci., 32, 233-242, doi:10.1175/1520-0469(1975)032<0233:TGMAAT >2.0.CO;2.

_ and P. J. Valdes, 1990: On the existence of storm tracks. J. Atmos. Sci., 47, 1854-1864, doi:10.1175/1520-0469(1990)047<1854: OTEOST $>2.0 . \mathrm{CO} ; 2$

Lee, S., and I. M. Held, 1993: Baroclinic wave packets in models and observations. J. Atmos. Sci., 50, 1413-1428, doi:10.1175/ 1520-0469(1993)050<1413:BWPIMA $>2.0$.CO;2.

Martius, O., C. Schwierz, and H. C. Davies, 2008: Far-upstream precursors of heavy precipitation events on the Alpine south-side. Quart. J. Roy. Meteor. Soc., 134, 417-428, doi:10.1002/qj.229.

Press, W. H., S. A. Teukolsky, W. T. Vetterling, and B. P. Flannery, 1996: Numerical Recipes in Fortran 90: The Art of Parallel Scientific Computing. Cambridge University Press, 1486 pp.

Shapiro, M. A., and A. J. Thorpe, 2004: THORPEX international science plan. WMO/ TD-1246, WWRP/ THORPEX 2, WMO, 51 pp. [Available online at http://www.wmo.int/pages/prog/arep/wwrp/ new/documents/CD_ROM_international_science_plan_v3.pdf.]

Souders, M. B., B. A. Colle, and E. K. M. Chang, 2014: A description and evaluation of an automated approach for featurebased tracking of Rossby wave packets. Mon. Wea. Rev., 142, 3505-3527, doi:10.1175/MWR-D-13-00317.1.

Swanson, K. L., 2007: Storm track dynamics. The Global Circulation of the Atmosphere, T. Schneider and A. H. Sobel, Eds., Princeton University Press, 78-103.

Wernli, H., M. Paulat, M. Hagen, and C. Frei, 2008: SAL-A novel quality measure for the verification of quantitative precipitation forecasts. Mon. Wea. Rev., 136, 4470-4487, doi:10.1175/ 2008MWR2415.1.

Wirth, V., 2001: Cyclone-anticyclone asymmetry concerning the height of the thermal and the dynamical tropopause. J. Atmos. Sci., 58, 2637, doi:10.1175/1520-0469(2001)058<0026:CAACTH >2.0.CO;2.

_ - and J. Eichhorn, 2014: Long-lived Rossby wave trains as precursors to strong winter cyclones over Europe. Quart. J. Roy. Meteor. Soc., 140, 729-737, doi:10.1002/qj.2191.

Zimin, A. V., I. Szunyogh, D. J. Patil, B. R. Hunt, and E. Ott, 2003: Extracting envelopes of Rossby wave packets. Mon. Wea. Rev., 131, 1011-1017, doi:10.1175/1520-0493(2003)131<1011: EEORWP $>2.0 . \mathrm{CO} ; 2$.

,-- , B. R. Hung, and E. Ott, 2006: Extracting envelopes of nonzonally propagating Rossby wave packets. Mon. Wea. Rev., 134, 1329-1333, doi:10.1175/MWR3122.1. 